\title{
Surgical Safety Checklist: Improved Patient Safety through Effective Teamwork
}

Chris Hayes

\section{Introduction and Background}

I recently had the opportunity to work in the kitchen of one of the top-rated restaurants in Canada. In addition to satisfying a culinary dream, the experience allowed me to observe a highfunctioning team outside of healthcare and reflect on attributes contributing to this team's success. Let's set the stage: This was a stressful and productive environment, focused on delivering high-quality and reliable outcomes. There were many team players, all with their unique set of roles and responsibilities and all working toward a common goal - to satisfy the customer. Hierarchy was present, with different grades of expertise and authority, and the executive chef orchestrating the group toward his expectations of perfection. We have seen this orchestration on food network television - often from a tyrannical leader; however, in the case of this top performer, the team members were respected for their skill sets, encouraged to excel, given opportunity to develop and provided with valuable feedback. Although the tyrannical approach can produce high quality, it comes at the cost of increased staff turnover, workplace stress and dissatisfaction and unnecessary variability and error (Donaldson-Feilder et al. 2011; Lloyd 2009).

Jump now to the aviation industry. Although considered an exemplar of a high-reliability organization, its team approach to safety was not always as central as it is today (Salas et al. 2010). Prior to the 1990s, the primary focus in pilot training programs was on individual development of technical skills, with little education on team development within the cockpit (Salas et al. 2010). Although the flight crew performed safety checklists, they did so independently. After several high-profile commercial airline accidents in the 1970s, root cause analysis of flight recorder data revealed that human factors and team dynamics were the cause, not equipment failure (Stone and Babcock 1998). As a result, the airline industry developed and introduced its first Cockpit Resource Management (CRM) program into its training in 1979 (Helmreich 2000). CRM teaches teams to use all available resources (equipment and people) to achieve safe flight operations. Initially, CRM was offered only to captains, with the intent of fostering open communication in the cockpit and flattening hierarchy; however, it was expanded to all flight crew members in the 1980s upon learning that there remained a reluctance among other crew members to "speak up," contributing to airline accidents (Helmreich 1992). As such, $C R M$ was changed to stand for Crew Resource Management. Although, there is no controlled evidence showing that CRM has resulted in the aviation industry's ultra-safe status, most aviation sectors have seen a significant reduction in accident rates since its introduction (Diehl 2001; Helmreich 2000).

Whether the culinary or aviation industry, there is general consensus that high-performing teams produce more reliable and quality outcomes (Baker et al. 2006). A team is defined as "two or more individuals, who have specific roles, perform interdependent tasks, are adaptable and share a common goal" (Baker 
et al. 2006: 1578). However, since teamwork is more than task work, merely forming a group with the above characteristics does not ensure that a team will function effectively. Teamwork is defined as a "set of interrelated knowledge, skills and attitudes that facilitate coordinated performance," and effective teams are those that demonstrate a shared commitment to that set (Baker et al. 2006: 1579). Within healthcare, it is well known that communication failures and ineffective teamwork have led to patient safety incidents and patient harm (Sutcliffe et al. 2004). In a review of over 3,500 reported sentinel events (those resulting in death or severe injury), the Joint Commission (2004) in the United States determined that the root causes were failures in communication and teamwork/training in over $60 \%$ and $50 \%$ of cases, respectively. Furthermore, a breakdown in communication was highlighted as the number one contributor to cases of wrong site surgery (Joint Commission 2001). Given the prevalence of adverse events related to surgery (Baker et al. 2004) and the huge number of surgical procedures performed annually, in 2008, the World Health Organization (WHO) launched its second Global Patient Safety Challenge, Safe Surgery Saves Lives (SSSL), in an attempt to reduce the morbidity and mortality associated with surgical complications (WHO 2009).

\section{The Surgical Safety Checklist}

This brings us to the now well-known Surgical Safety Checklist (SSC; WHO 2009). With evidence underscoring communication issues in surgery (Lingard et al. 2004) and the positive impacts of checklists in aviation and medicine (Lingard et al. 2008; Pronovost et al. 2006), eight global pilot sites (one Canadian) participated in a study to evaluate the effect of a three-phase safety checklist, to be completed prior to the administration of anesthetic, prior to incision and before the patient leaves the operating room (Haynes et al. 2009). Using a pre-post design, a study following 8,000 surgical procedures found that the implementation of the SSC resulted in a 30\% reduction in the rates of both surgical complications and deaths (Haynes et al. 2009). Furthermore, there was an observed increase in the delivery of six surgical best practices. The publication of this article was met with much media attention globally, along with some criticism. Headlines proclaimed that a simple paper checklist can save lives, leaving the public shocked that such a practice was not already in place. The study design was criticized for its non-controlled nature and failure to describe which elements of the checklist were responsible for the observed changes. Nevertheless, there was immediate worldwide interest in embedding this "simple tool" into peri-operative practice. The UK National Health Service (NHS) declared the completion of the checklist mandatory less than one month following the article's publication (personal observation), and in Canada public reporting of checklist completion was made mandatory in July 2010 (Ministry of Health and Long-Term Care
[MOHLTC] 2009) and became an Accreditation Canada required organizational practice in January 2011 (Accreditation Canada n.d.).

\section{Introducing the SCC in Canada}

Recognizing the impact that the SSC could have on patient safety, the Canadian Patient Safety Institute (CPSI) formed and supported the National Safe Surgery Saves Lives Working Group in August 2008, with a membership representing nursing, surgery, anesthesia and national professional and accreditation organizations, as well as aviation, human factors and cognitive psychology. Instrumental to the group was the recruitment of Dr. Bryce Taylor, former surgeon-in-chief at Toronto's University Health Network, as special advisor given his expertise with the implementation of the SSC. The overall mandate of the Working Group was to lead further development, adaptation (see Figure 1), implementation and support for the SSSL Campaign within the Canadian context. The Working Group coordinated national workshops, keynote presentations at national conferences, rounds at local hospitals, virtual grand rounds, a virtual collaborative and a mentor/support network. Within the first year, 5,000 checklist implementation kits were downloaded (CPSI 2010) and 67\% of hospitals reported using the three-part checklist (Flintoft July 2011, personal communication). As of June 2011, Ontario hospitals were reporting the completion of the SSC in 99\% of surgeries (MOHLTC 2011).

During the Canadian SSSL Campaign rollout, much feedback was obtained from participating organizations. Hospitals reported the enthusiastic adoption of the SSC by their surgical teams and the emergence of checklist champions within anaesthesia, nursing and surgery. Anecdotal reports surfaced of improved teamwork dynamics in the operating room, a sense of improved patient safety and examples of patient harm being averted through the identification of safety issues during checklist completion. This response is in keeping with the findings of others. Participants in the WHO checklist study reported a perceived increase in teamwork and safety climate following the implementation of the SSC (Haynes et al. 2011). Taylor et al. (2010) surveyed operating room staff and found a perceived improvement in communication, teamwork, respect and patient safety related to the implementation of the SSC, a phenomenon that was also detected later through the responses of operating room staff on a hospital-wide employee opinion survey. Improved patient outcomes following implementation of surgical safety checklists has been clearly demonstrated within the Veterans Affairs (Neily et al. 2010) as well as in the Netherlands (de Vries et al. 2010) and Iran (Askarian et al. 2011).

Despite the positive feedback during the Canadian SSSL Campaign, there was greater feedback about the challenges that hospitals and surgical teams faced in implementing the SSC. Concerns were voiced about gaining buy-in, about whose role 
it was to complete the checklist and that the checklist would cause surgical delays and possible cancellations (although there is evidence showing the contrary [Ali et al. 2011; Nundy et al. 2008]). Also there were challenges in having all the team members present during the three phases of the checklist; teams adopted a "tick-and-flick" attitude and did not really discuss the checklist elements; there was also a sense of pressure to complying with provincial and accreditation requirements. Similar challenges were experienced during the rollout of the SSC in the United Kingdom (Vats et al. 2010). Hierarchy, timing logistics, the perception of work duplication and the relevance of the checklist were factors that either inhibited its adoption or resulted in the checklist process being incomplete and hurried and the participants being dismissive or absent (Vats et al. 2010).

\section{Overcoming Barriers to Implementation}

With growing evidence demonstrating the effectiveness of the SSC in improving patient safety and outcomes and the knowledge of the challenges to its implementation, the question that arose was, what strategies can be used to increase the likelihood of successful and sustainable adoption of the checklist into peri-operative practice and surgical culture? Fortunately, lessons from those organizations that have embedded the checklist as a standard operating procedure can be used as guides for others. In anecdotes from the Canadian SSSL Campaign and strategies reported in the literature, there are common elements that are associated with the successful implementation of the SSC (by CPSI and WHO) that have been adapted from other change management strategies (Kotter 1996; Reinertsen et al. 2007).

\section{Leadership Support}

As with all quality improvement plans, leadership support for the implementation of the SSC has been paramount. Leadership support was deemed the strongest independent predictor of successful checklist implementation among 64 Veterans Health Administration facilities (Paull et al. 2009). In studying the implementation efforts of five hospitals, Conley et al. (2011) found that the engagement of leadership was seen as a key factor in the success of the checklist adoption. Having the department chiefs as members of the implementation team and actively promoting the SSC was deemed most successful, whereas having clinical leadership merely support the idea but not show active involvement was seen as less effective (Conley et al. 2011).

\section{Timing of Mandatory Participation}

The messaging from leadership is also important. Strategies that involved an upfront or early mandatory approach to SSC checklist adoption have induced resistance. At the European launch of the SSSL Campaign, following presentations from the keynote speakers, NHS informed all delegates that the checklist would immediately be mandatory, require provider signatures and be audited (personal observation). This quickly turned the atmosphere from one of eager participation to vocal opposition. Others have commented on the tactic of a mandated checklist (Schlack and Boermeester 2010; Taylor et al. 2010; WHO 2009), supporting the strategy of upfront willing engagement from providers with enforcement as a later-stage approach. This has been felt to maintain the integrity of the checklist process and avoid the tick-and-flick approach (Taylor et al. 2010).

\section{Implementation Team and Approach}

Other pivotal factors guiding the success of SSC adoption are the formation of an implementation team and the approach that they use (Safer Healthcare Now! n.d., WHO 2009). Given the interdisciplinary nature of the SSC, having all disciplines, particularly anesthesia and surgery, actively involved is important as they are likely to successfully influence their peers (Hayes et al. 2010; Paull et al. 2009; Reinertsen et al. 2007). This is especially true if physician champions are recruited as team members. Such champions act as vocal supporters and demonstrate the model process for checklist completion. A key tenet of quality improvement, supported by feedback from Canadian hospitals reporting successful checklist adoption (Flintoft, July 2011, personal communication), is to start the implementation process on a small scale - on one surgical team or in one division. This is important for several reasons: it allows for more in-depth training - to demonstrate and master the "correct" way of completing the checklist (Taylor et al. 2010); permits feedback from the front line in modifying the checklist content and process to the local environment; provides the opportunity to openly discuss barriers; and is more likely to gain team buy-in and to foster champions (Langley et al. 2009). Subsequent scale up is then often easier as word of the successful implementation spreads to other teams.

\section{Burning Platform}

Another tenet of quality improvement and change management is the use of data to create a "burning platform" (Kotter 1996; Langley et al. 2009). Although published outcome data on the effectiveness of the SSC may help set the stage and define its purpose, it is often local data that drive practice change (Hysong 2009; Safer Healthcare Now! n.d.). Sources of local data that can be used to support the implementation process include the following: (1) prospective audit feedback of surgical cases to identify opportunities in which the use of the checklist could have prevented error or harm, (2) a retrospective review of surgical cases in which the consequences could have been altered by checklist use and (3) a prospective collection of "good catches" during the initial rollout stage as proof of the checklist's utility. Sharing of these data and stories helps build the case for checklist adoption. Another important use of data is in sustaining checklist use. Ongoing observation and audit of the checklist process can continue to identify good catches and ensure correct check- 
FIGURE 1.

Surgical Safety Checklist - Canadian Version for reference only

\begin{tabular}{|c|c|c|}
\hline $\operatorname{cPSI}$ & $\begin{array}{l}\text { SURGICAL SAFETY } \\
\text { CHECKLIST } \\
\text { www.safesurgerysaveslives.ca }\end{array}$ & $\begin{array}{l}\text { Your } \\
\text { Organizational } \\
\text { Logo }\end{array}$ \\
\hline 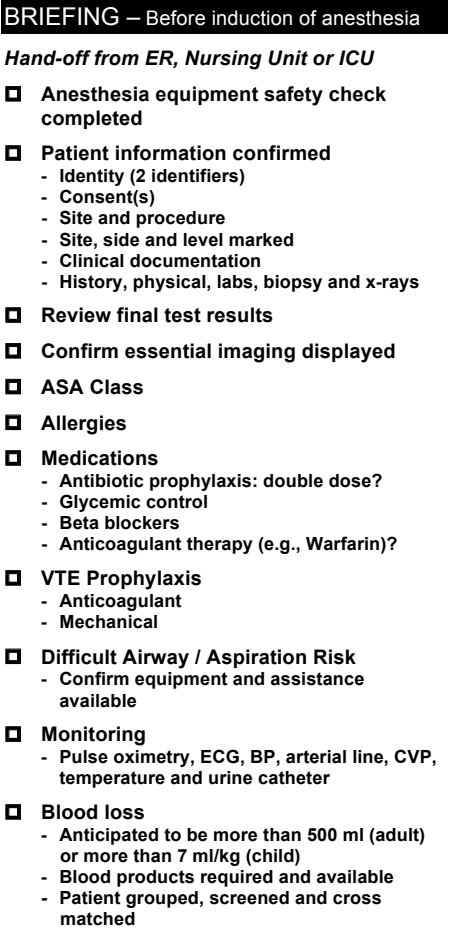 & 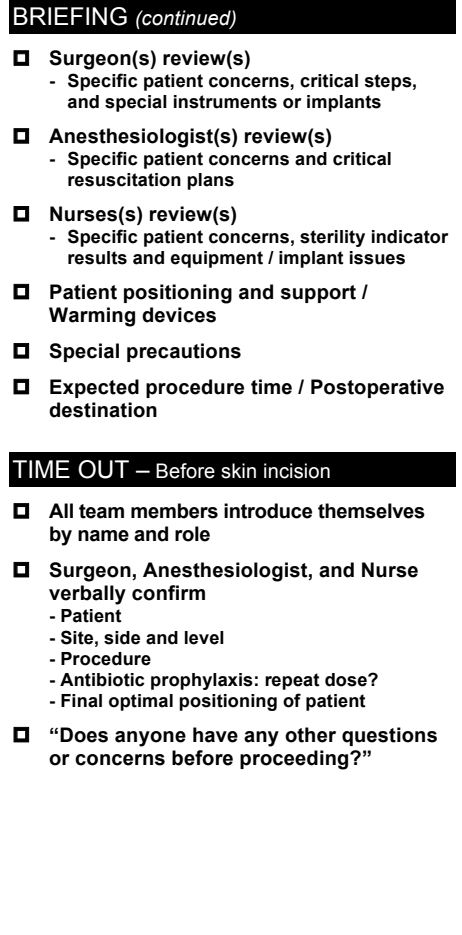 & 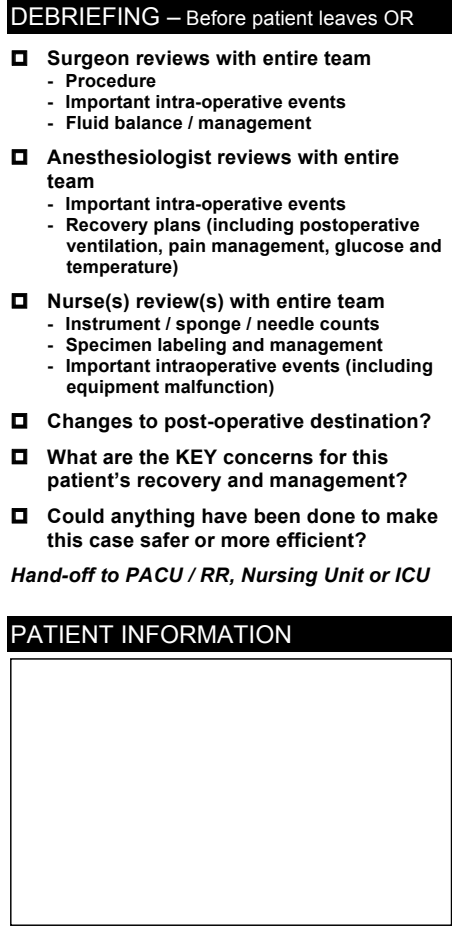 \\
\hline
\end{tabular}

list performance. As described above, several studies have used pre-post survey techniques to assess the attitudinal and culture shift associated with surgical safety checklist use (Haynes et al. 2011; Taylor et al. 2010), a strategy that can be employed locally for the purpose of providing feedback and securing sustainability.

\section{Team Training}

Notwithstanding the impact that the SSC has had and will have on patient safety outcomes, it is merely a communication tool to remind teams to address key safety issues prior to and during surgery. In the majority of reported studies that evaluated surgical safety checklists, team training was included in the intervention (de Vries et al. 2010; Haynes et al. 2009; Neily et al. 2010; Taylor et al. 2010). Furthermore, safety culture and team perceptions were usually addressed, with demonstrable improvement realized post-implementation of the checklist (Haynes et al. 2009; Neily et al. 2010).
So, is the SSC a mechanism for introducing team training and safety culture awareness into surgical care? Similar to aviation, it was the use of checklists coupled with widespread team training that transformed the industry to its ultra-safe status (Helmreich 2000). For example, the primary focus of the program at the Veterans Health Administration was to implement an interprofessional medical team training program into hospitals providing surgical services; a safety checklist was introduced after program completion (Neily et al. 2010). The program involved a two-month preparatory session with each site's implementation team, followed by a day-long onsite learning session. Using CRM-based theory, the inter-professional participants were trained to work as teams, challenge each other when safety risks were identified, perform situational awareness checks and learn rules of conduct for communication. To allow staff to attend as full teams, the operating rooms were closed for the day (a clear demonstration of leadership commitment to the program). The 
program resulted in overall increased perceptions of teamwork and communication, an increased use of surgical safety checklists and an $18 \%$ reduction in mortality (Neily et al. 2010). The authors proposed that the team training facilitated more open communication in the operating rooms and that the checklist guided the discussion toward improved safety outcomes (Neily et al. 2010). Another widely used and evidence-based program for team training is the TeamSTEPPS program developed by the US Department of Defense in collaboration with the Agency for Healthcare Research and Quality (King et al. 2008). Similar to the training in the Veterans Health Administration program, TeamSTEPPS trains inter-professional teams in the importance of leadership, situational awareness, mutual support and effective communication (King et al. 2008). The implementation of this program into surgical programs has also resulted in improved team performance and outcomes (Armour Forse et al. 2011; Capella et al. n.d.).

Despite the impressive literature on communication issues in Canadian operating rooms (for example, Lingard et al. 2004), there are no published data of the effective implementation of inter-professional surgical team training in Canada. However, there is good reason to believe that programs similar to that of the Veterans Health Administration and TeamSTEPPS could be developed and implemented in Canadian hospitals and residency programs, given the similarities in sites included in those studies as well as in surgical team structures and processes. Following this logic, there is also good reason to implement inter-professional team training into health professional training programs. This would enable surgical team members to apply the required knowledge, skills and attitudes during their training rather than learning how to work in teams after the fact. Such inclusion would meet the competency-based requirements and recommendations of the American Council of Graduate Medical Education, the Royal College of Physicians and Surgeons of Canada and the CPSI Patient Safety Competencies (Accreditation Council for Graduate Medical Education n.d.; Frank and Brien 2008; Royal College of Physicians and Surgeons of Canada 2011).

\section{Conclusion}

Equipping healthcare providers with the training to function as high-performing teams and providing them with tools to improve communication and patient safety may be the recipe for reliable improvements in safety culture and patient outcomes in surgery (and likely many other medical domains). Although more conclusive proof on the benefits of widespread inter-professional team training may be warranted, there is significant evidence to warrant its inclusion into surgical, anaesthesia and operating room nursing training programs and practice settings. $\mathrm{HQ}$

\section{Acknowledgements}

With thanks to the members of the National Working Group: Rob Barrett, Paula Beard, Douglas Bell, Bill Berry, Peter Blair, Cecilia Bloxom, Craig Bosenberg, Susan Brien, Donna Davis, Lizzie Edmondson, Virginia Flintoft, Norma Freeman, Paul Hendry, Sonia Hill, Gregory Kennedy, Deon Louw, Cindy Macfarlane, Anne MacLaurin, Bonnie McLeod, Marie Owen, Anna Pevreal, Ioana Popescu, Melanie Rathgeber, Tanis Rolefstad, Bryce Taylor, Jason Thompson, Marlies Van Dijk, Stan Vuksic and Daniel Wong

\section{References}

Accreditation Canada n.d. Required Organizational Practices. Ottawa, ON: Accreditation Canada. Retrieved Sept. 2011. <http://www. accreditation.ca/accreditation-programs/qmentum/required-organizational-practices/>.

Accreditation Council for Graduate Medical Education. n.d. ACGME Outcome Project. Chicago, IL: Author. Retrieved October 14, 2011. <http://www.acgme.org/outcome/comp/compFull.asp>.

Ali, M., A. Osborne, R. Bethune and A. Pullyblank. 2011. "Preoperative Surgical Briefings Do Not Delay Operating Room Start Times and Are Popular with Surgical Team Members." Journal of Patient Safety 7(3): 139-43. DOI: 10.1097/PTS.0b013e31822a9fbc.

Armour Forse, R., J.D. Bramble and R. McQuillan. 2011. "Team Training Can Improve Operating Room Performance.” Surgery 150(4): 771-78. DOI: 10.1016/j.surg.2011.07.076.

Askarian, M., F. Kouchak and C.J. Palenik. 2011. "Effect of Surgical Safety Checklists on Postoperative Morbidity and Mortality Rates, Shiraz, Faghihy Hospital, a 1-Year Study." Quality Management in Health Care 20(4): 293-97. DOI: 10.1097/QMH.0b013e318231357c.

Baker, D.P, R. Day and E. Salas. 2006. "Teamwork as an Essential Component of High-Reliability Organizations." Health Services Research 41(4, Pt. 2): 1576-98. DOI: 10.1111/j.1475-6773.2006.00566.x.

Baker, G.R., P.G. Norton, V. Flintoft, R. Blais, A. Brown, J. Cox et al. 2004. "The Canadian Adverse Events Study: The Incidence of Adverse Events among Hospital Patients in Canada." Canadian Medical Association Journal 170(11): 1678-86.

Canadian Patient Safety Institute. 2010. [Data regarding downloading of Surgical Safety Checklist.] Unpublished raw data.

Capella, J., S. Smith, A. Philp, T. Putnam, C. Gilbert, W. Fry et al. n.d. "Teamwork Training Improves the Clinical Care of Trauma Patients." Journal of Surgical Education 67(6): 439-43. DOI: 10.1016/j. jsurg.2010.06.006.

Conley, D.M., S.J. Singer, L. Edmondson, W.R. Berry and A.A. Gawande. 2011. "Effective Surgical Safety Checklist Implementation." Journal of the American College of Surgeons 212(5): 873-79. DOI: 10.1016/j.jamcollsurg.2011.01.052.

de Vries, E.N., H.A. Prins, R.M.P.H. Crolla, A.J. den Outer, G. van Andel, S. van Helden et al. 2010. "Effect of a Comprehensive Surgical Safety System on Patient Outcomes." New England Journal of Medicine 363(20): 1928-37. DOI: 10.1056/NEJMsa0911535.

Diehl, A. 2001. "Culture, Environment, and CRM.” In T. Kern, ed., Culture, Environment, and CRM. New York: McGraw-Hill Professional.

Donaldson-Feilder, E., R. Lewis and J. Yarker. 2011. Preventing Stress in Organizations: How to Develop Positive Managers. Malden, MA: John Wiley and Sons. 
Frank, J. and S. Brien, eds. 2008. The Safety Competencies: Enhancing Patient Safety Across the Health Professions. Ottawa, ON: Canadian Patient Safety Institute.

Hayes, C., V. Yousefi, T. Wallington and A. Ginzburg. 2010. "Case Study of Physician Leaders in Quality and Patient Safety, and the Development of a Physician Leadership Network." Healthcare Quarterly 13(Special Number): 68-73.

Haynes, A.B., T.G. Weiser, W.R. Berry, S.R. Lipsitz, A.H. Breizat, E.P. Dellinger et al. 2011. "Changes in Safety Attitude and Relationship to Decreased Postoperative Morbidity and Mortality following Implementation of a Checklist-Based Surgical Safety Intervention." BMJ Quality and Safety 20(1): 102-7.

Haynes, A.B, T.G. Weiser, W.R. Berry, S.R. Lipsitz, A.-H.S. Breizat, E.P. Dellinger et al. 2009. "A Surgical Safety Checklist to Reduce Morbidity and Mortality in a Global Population." New England Journal of Medicine 360(5): 491-99. DOI: 10.1056/NEJMsa0810119.

Helmreich, R. 1992. Human Factors Aspects of the Air Ontario Crash at Dryden. Toronto, ON: Minister of Supply and Services.

Helmreich, R.L. 2000 " On Error Management: Lessons from Aviation." BMJ 320(7237): 781-85. DOI: 10.1136/bmj.320.7237.781.

Hysong, S.J. 2009. "Meta-Analysis: Audit and Feedback Features Impact Effectiveness on Care Quality." Medical Care 47(3): 356-63. DOI: 10.1097/MLR.0b013e3181893f6b.

Joint Commission. 2001. A Follow-Up Review of Wrong Site Surgery. Oakbrook Terrace, IL: Author. Retrieved September 2011. <http:// www.jointcommission.org/assets/1/18/SEA_24.pdf>.

Joint Commission. 2004. Improving America's Hospitals - The Joint Commission's Annual Report on Quality and Safety 2003. Oakbrook Terrace, IL: Author.

King, H.B., J. Battles, D.P. Baker, A. Alonso, E. Salas, J. Webster et al. 2008. "TeamSTEPPS: Team Strategies and Tools to Enhance Performance and Patient Safety." In K. Henriksen, J.B. Battles, M.A. Keyes and M.L. Grady, eds., Advances in Patient Safety: New Directions and Alternative Approaches (Vol. 3: Performance and Tools). Rockville, MD: Agency for Healthcare Research and Quality.

Kotter, J.P. 1996. Leading Change. Boston, MA: Harvard Business Press.

Langley, G.J., R. Moen, K.M. Nolan, T.W. Nolan, C.L. Norman and L.P. Provost. 2009. The Improvement Guide: A Practical Approach to Enhancing Organizational Performance. San Francisco, CA: John Wiley and Sons.

Lingard, L., G. Regehr, B. Orser, R. Reznick, G.R. Baker, D. Doran et al. 2008. "Evaluation of a Preoperative Checklist and Team Briefing among Surgeons, Nurses, and Anesthesiologists to Reduce Failures in Communication." Archives of Surgery 143(1): 12-17, discussion 18. DOI: 10.1001/archsurg.2007.21.

Lingard, L., S. Espin, S. Whyte, G. Regehr, G.R. Baker, R. Reznick et al. 2004. "Communication Failures in the Operating Room: An Observational Classification of Recurrent Types and Effects." Quality and Safety in Health Care 13(5): 330-34. DOI: 10.1136/qhc.13.5.330.

Lloyd, T. 2009. Business at a Crossroads: The Crisis of Corporate Leadership. New York: Palgrave Macmillan.

Ministry of Health and Long-Term Care. 2009. Hospitals to Use Surgical Safety Checklist. Toronto, ON: Author. <http://www.health.gov.on.ca/ en/news/release/2009/oct/patientsafety_1year_nr_20090922_2_. pdf>. Retrieved Sept 2011.

Ministry of Health and Long-Term Care. 2011. Patient Safety Indicator Reporting: Ontario Totals. Toronto, ON: Author. Retrieved September 2011. <http://patientsafetyontario.net/Reporting/en/ PSIR_ProvincialIndicatorReporting.aspx? View $=1 \&$ hosptid $=1444 \&$ s eltype $=4 \&$ str $=$ s\# $>$.
Neily, J., P.D. Mills, Y. Young-Xu, B.T. Carney, P. West, D.H. Berger et al. 2010. Association between Implementation of a Medical Team Training Program and Surgical Mortality." Journal of the American Medical Association 304(15): 1693-700. DOI: 10.1001/ jama.2010.1506.

Nundy, S., A. Mukherjee, J.B. Sexton, P.J. Pronovost, A. Knight, L.C. Rowen et al. 2008. "Impact of Preoperative Briefings on Operating Room Delays: A Preliminary Report." Archives of Surgery 143(11): 1068-72. DOI: 10.1001/archsurg.143.11.1068.

Paull, D.E., L.M. Mazzia, B.S. Izu, J. Neily, P.D. Mills and J.P. Bagian. 2009. "Predictors of Successful Implementation of Preoperative Briefings and Postoperative Debriefings after Medical Team Training." American Journal of Surgery 198(5): 675-78. DOI: 10.1016/j. amjsurg.2009.07.008.

Pronovost, P., D. Needham, S. Berenholtz, D. Sinopoli, H. Chu, S. Cosgrove et al. 2006. "An Intervention to Decrease Catheter-Related Bloodstream Infections in the ICU." New England Journal of Medicine 355(26): 2725-32. DOI: 10.1056/NEJMoa061115.

Reinertsen, J.L., A.G. Gosfield, W. Rupp and J.W. Whittington. 2007. Engaging Physicians in a Shared Quality Agenda (IHI Innovation Series White Paper). Cambridge, MA: Institute for Healthcare Improvement.

Royal College of Physicians and Surgeons of Canada. 2011. Competence by Design: Royal College White Paper Series. Ottawa, ON: Author. Retrieved October 14, 2011. <http://rcpsc.medical.org/residency/ fmec/index.php>.

Safer Healthcare Now! n.d. Safe Surgery Saves Lives. Edmonton, AB: Canadian Patient Safety Institute. Retrieved October 14, 2011. $<$ http://www.saferhealthcarenow.ca/en/interventions/safesurgery/ pages/default.aspx $>$.

Salas, E., N.J. Cooke and J.C. Gorman. 2010. “The Science of Team Performance: Progress and the Need for More ..." Human Factors 52(2): 344-46.

Schlack, W.S. and M.A. Boermeester. 2010. "Patient Safety during Anaesthesia: Incorporation of the WHO Safe Surgery Guidelines into Clinical Practice." Current Opinion in Anaesthesiology 23(6): 754-58. DOI: 10.1097/ACO.0b013e3283400b26.

Stone, R. and G.L. Babcock. 1998. “Airline Pilot's Perspective.” In E. Wiener and D. Nagel, eds., Human Factors in Aviation. San Diego, CA: Academic Press.

Sutcliffe, K.M., E. Lewton and M.M. Rosenthal. 2004. "Communication Failures: An Insidious Contributor to Medical Mishaps.” Academic Medicine 79(2): 186-94.

Taylor, B., A. Slater and R. Reznick. 2010. "The Surgical Safety Checklist Effects Are Sustained, and Team Culture Is Strengthened." The Surgeon 8(1): 1-4.

Vats, A., C.A. Vincent, K. Nagpal, R.W. Davies, A. Darzi and K. Moorthy. 2010. "Practical Challenges of Introducing WHO Surgical Checklist: UK Pilot Experience.” BMJ 340: b5433.

World Health Organization. 2009. Safe Surgery Saves Lives. Geneva, Switzerland: Author. Retrieved October 14, 2011. <http://www.who. int/patientsafety/safesurgery/en/index.html>.

\section{About the Authors}

Chris W. Hayes, MD, MSc, MEd, FRCPC, is assistant professor, Department of Medicine, University of Toronto, medical officer of the Canadian Patient Safety Institute and medical director of Quality and Patient Safety, St. Michael's Hospital, Toronto. 